\title{
Fluctuations in a coupled-oscillator model of the cardiovascular system
}

\author{
Jorge A. González ${ }^{a}$, Jose J. Suárez-Vargas ${ }^{b}$, \\ Aneta Stefanovska ${ }^{b}$ and Peter V. E. McClintock ${ }^{b}$; \\ ${ }^{a}$ Physics Center, Venezuelan Institute for Scientific Research, \\ Km. 11, Carretera Panamericana, Caracas 1020-A, Venezuela; \\ ${ }^{b}$ Department of Physics, Lancaster University, Lancaster LA1 4YB, UK;
}

\begin{abstract}
We present a model of the cardiovascular system (CVS) based on a system of coupled oscillators. Using this approach we can describe several complex physiological phenomena that can have a range of applications. For instance, heart rate variability (HRV), can have a new deterministic explanation. The intrinsic dynamics of the HRV is controlled by deterministic couplings between the physiological oscillators in our model and without the need to introduce external noise as is commonly done. This new result provides potential applications not only for physiological systems but also for the design of very precise electronic generators where the frequency stability is crucial. Another important phenomenon is that of oscillation death. We show that in our CVS model the mechanism leading to the quenching of the oscillations can be controlled, not only by the coupling parameter, but by a more general scheme. In fact, we propose that a change in the relative current state of the cardiovascular oscillators can lead to a cease of the oscillations without actually changing the strength of the coupling among them. We performed real experiments using electronic oscillators and show them to match the theoretical and numerical predictions. We discuss the relevance of the studied phenomena to real cardiovascular systems regimes, including the explanation of certain pathologies, and the possible applications in medical practice.
\end{abstract}

Keywords: nonlinear coupled oscillators, oscillation death, heart rate variability, cardiovascular models, analogue simulations.

\section{INTRODUCTION}

Arrays of coupled oscillators are ubiquitous in nature and technology. ${ }^{1}$ The theory of coupled oscillators has been applied to arrays of lasers, Josephson junctions and to the explanation of phenomena related to biological rhythms. $^{2}$

Many papers have been dedicated to the synchronization effect. ${ }^{3}$ This occurs when the coupling strength exceeds a certain critical value. In this case, the amplitudes are not affected. For stronger couplings the amplitude dynamics is very important and new phenomena can occur. Among these phenomena there is the so-called "amplitude death" or "oscillation death" ${ }^{-7}$, in which the oscillators can collapse to a state of zero amplitude.

Recently there has been a wealth of work dedicated to oscillation death and similar phenomena in many different systems. ${ }^{8-12}$

In the present paper we investigate a mathematical model of the cardiovascular system based on an array of coupled oscillators. The basic unit in the model is an oscillator that possesses some mathematical features motivated by physiological understanding and by the analysis of measured time series ${ }^{13,14}$. Oscillators with linear couplings have been found to reproduce some of the main characteristic features of the experimental

Further author information:

J.A.G. : E-mail: jorge@ivic.ve,

J.J.S.V. : E-mail: jose.suarezvargas@lancaster.ac.uk,

A.S. : E-mail: aneta@lancaster.ac.uk,

P.V.E.M. : E-mail: p.v.e.mcclintock@lancaster.ac.uk 
observations $^{15}$. However, in previous papers ${ }^{15}$, the addition of noise was necessary for the explanation of the variability of cardiac and respiratory frequencies. Our results show that heart-rate variability can have a new deterministic explanation. Using techniques of dynamical systems theory, we show that the frequencies of the cardiac and respiratory oscillators can experience variabilities very similar to those observed in real measurements. The intrinsic dynamics of the cardiovascular system is controlled by deterministic coupling between the physiological oscillators and without any need to add external noise.

We also show that the phenomenon of oscillation death can occur in the model of cardiovascular system. Moreover, the mechanism leading to the quenching of the oscillators can be controlled, not only by the coupling parameters, but by a more general scheme. In fact, we propose that a change in the relative current state of the cardiovascular oscillators can lead to a cessation of the oscillations without actually changing the strength of the coupling among them. Furthermore, we show that the bifurcation route to oscillation death in our system can produce different non-zero fixed points. Hence, the oscillators can stop beating but actually keeping certain constant non-zero amplitude. We discuss also the results of real experiments using electronic oscillators.

\section{PRESENTATION OF THE MODEL}

The model of the cardiovascular system is based on a system of five coupled oscillators ${ }^{13,14}$. Their physiological functions are the following: the heart, respiration, myogenic activity, neurogenic activity and endothelial related metabolic activity. The basic unit in the model is the Poincaré oscillator:

$$
\begin{aligned}
\dot{x_{i}} & =-x_{i} q_{i}-y_{i} \omega_{i}+P_{i}(\mathbf{X}, \mathbf{Y}) \\
\dot{y_{i}} & =-y_{i} q_{i}+x_{i} \omega_{i}+Q_{i}(\mathbf{X}, \mathbf{Y})
\end{aligned}
$$

where $q_{i}=\alpha_{i}\left(\sqrt{\left(x_{i}^{2}+y_{i}^{2}\right)}-a_{i}\right), a_{i}, \omega_{i}=2 \pi f_{i}$ and $\alpha_{i}$ are constant parameters, and $P_{i}(\mathbf{X}, \mathbf{Y})$ and $Q_{i}(\mathbf{X}, \mathbf{Y})$ are coupling terms.

\section{OSCILLATION DEATH}

\subsection{Theoretical Background}

In this section we will consider that the interaction between the oscillators is a mean field term:

$$
\begin{aligned}
P_{i}(\mathbf{X}, \mathbf{Y}) & =\sum x_{j} \\
Q_{i}(\mathbf{X}, \mathbf{Y}) & =0
\end{aligned}
$$

Let us first introduce some concepts.

Suppose we are studying an autonomous dynamical system:

$$
\begin{aligned}
& \dot{z}_{1}=F_{1}\left(z_{1}, z_{2}, \ldots, z_{n}\right), \\
& \dot{z}_{2}=F_{2}\left(z_{1}, z_{2}, \ldots, z_{n}\right), \\
& \ldots \\
& \dot{z}_{n}=F_{n}\left(z_{1}, z_{2}, \ldots, z_{n}\right) .
\end{aligned}
$$

All the points (in phase space) that satisfy the equations

$$
\begin{gathered}
F_{1}\left(z_{1}, z_{2}, \ldots, z_{n}\right)=0 \\
F_{2}\left(z_{1}, z_{2}, \ldots, z_{n}\right)=0 \\
\ldots \\
F_{n}\left(z_{1}, z_{2}, \ldots, z_{n}\right)=0
\end{gathered}
$$


are called fixed points of the dynamical system (3). The stability and quality of the fixed points can be determined by the eigenvalues of the Jacobi matrix, provided their real parts are nonzero.

Given a fixed point $\mathbf{Z}^{0}=\left(z_{1}^{0}, z_{2}^{0}, \ldots, z_{n}^{0}\right)$, the eigenvalues of the Jacobi matrix corresponding to this fixed point can be calculated from the equation:

$$
\left|\begin{array}{cccc}
\frac{\partial F_{1}\left(\mathbf{Z}^{0}\right)}{\partial z_{1}}-\lambda & \frac{\partial F_{1}\left(\mathbf{Z}^{0}\right)}{\partial z_{2}} & \cdots & \frac{\partial F_{1}\left(\mathbf{Z}^{0}\right)}{\partial z_{n}} \\
\frac{\partial F_{2}\left(\mathbf{Z}^{0}\right)}{\partial z_{1}} & \frac{\partial F_{2}\left(\mathbf{Z}^{0}\right)}{\partial z_{1}}-\lambda & \cdots & \frac{\partial F_{2}\left(\mathbf{Z}^{0}\right)}{\partial z_{n}} \\
\vdots & \vdots & & \vdots \\
\frac{\partial F_{n}\left(\mathbf{Z}^{0}\right)}{\partial z_{1}} & \frac{\partial F_{n}\left(\mathbf{Z}^{0}\right)}{\partial z_{2}} & \cdots & \frac{\partial F_{n}\left(\mathbf{Z}^{0}\right)}{\partial z_{n}}-\lambda
\end{array}\right|=0 .
$$

\subsection{Two Oscillators}

$$
\begin{aligned}
& \dot{x_{1}}=-x_{1} \alpha\left(\sqrt{x_{1}^{2}+y_{1}^{2}}-a_{1}\right)-y_{1} \omega_{1}+\epsilon\left(x_{1}+x_{2}\right), \\
& \dot{y_{1}}=-y_{1} \alpha\left(\sqrt{x_{1}^{2}+y_{1}^{2}}-a_{1}\right)+x_{1} \omega_{1}, \\
& \dot{x_{2}}=-x_{2} \alpha\left(\sqrt{x_{2}^{2}+y_{2}^{2}}-a_{2}\right)-y_{2} \omega_{2}+\epsilon\left(x_{1}+x_{2}\right), \\
& \dot{y_{2}}=-y_{2} \alpha\left(\sqrt{x_{2}^{2}+y_{2}^{2}}-a_{2}\right)+x_{2} \omega_{2} .
\end{aligned}
$$

The point $(0,0,0,0)$ is always a fixed point. For $\epsilon=0$, this point is unstable (focus or node). Besides this fixed point, there is the well-known stable limit cycle, which is the reason why this system can be considered an oscillator model. The eigenvalues corresponding to this fixed point when $\epsilon \neq 0$ can be calculated using equation $(5)$ :

$$
\left|\begin{array}{cccc}
\left(\alpha a_{1}+\epsilon\right)-\lambda & -\omega_{1} & \epsilon & 0 \\
\omega_{1} & \alpha a_{1}-\lambda & 0 & 0 \\
\epsilon & 0 & \left(\alpha a_{2}+\epsilon\right)-\lambda & -\omega_{2} \\
0 & 0 & \omega_{2} & \alpha a_{2}-\lambda
\end{array}\right|=0
$$

The origin can become a stable fixed point and the limit cycle does not exist anymore if the following conditions are satisfied:

$$
\begin{aligned}
& 2 \alpha a_{1}+\epsilon<0, \\
& 2 \alpha a_{2}+\epsilon<0,
\end{aligned}
$$

For instance, if $a_{2}>a_{1}$, then the condition $\epsilon<-2 \alpha a_{2}$ is sufficient for the amplitude death to occur.

When $\epsilon>0$, there is a critical value $\epsilon_{c}$ such that, for $\epsilon>\epsilon_{c}$, four new fixed points can appear: two unstable and two asymptotically stable. The Fig. 1 shows the bifurcation diagram for the four variables $x_{1}, y_{1}, x_{2}, y_{2}$. Notice that the origin is always an unstable fixed point. The end of the oscillations is marked by the appearance of the new fixed points at $\epsilon \sim 3.578$.

The new fixed points are obtained from the set of algebraic equations.

$$
\begin{array}{lll}
-x_{1} \alpha\left(\sqrt{x_{1}^{2}+y_{1}^{2}}-a_{1}\right)-y_{1} \omega_{1}+\epsilon\left(x_{1}+x_{2}\right) & =0, \\
-y_{1} \alpha\left(\sqrt{x_{1}^{2}+y_{1}^{2}}-a_{1}\right)+x_{1} \omega_{1} & =0, \\
-x_{2} \alpha\left(\sqrt{x_{2}^{2}+y_{2}^{2}}-a_{2}\right)-y_{2} \omega_{2}+\epsilon\left(x_{1}+x_{2}\right) & =0,
\end{array}
$$

After some algebra, we get the relations:

$$
\begin{aligned}
& \omega_{1}\left(x_{1}^{2}+y_{1}^{2}\right)=\epsilon y_{1}\left(x_{1}+x_{2}\right), \\
& \omega_{2}\left(x_{2}^{2}+y_{2}^{2}\right)=\epsilon y_{2}\left(x_{1}+x_{2}\right) .
\end{aligned}
$$



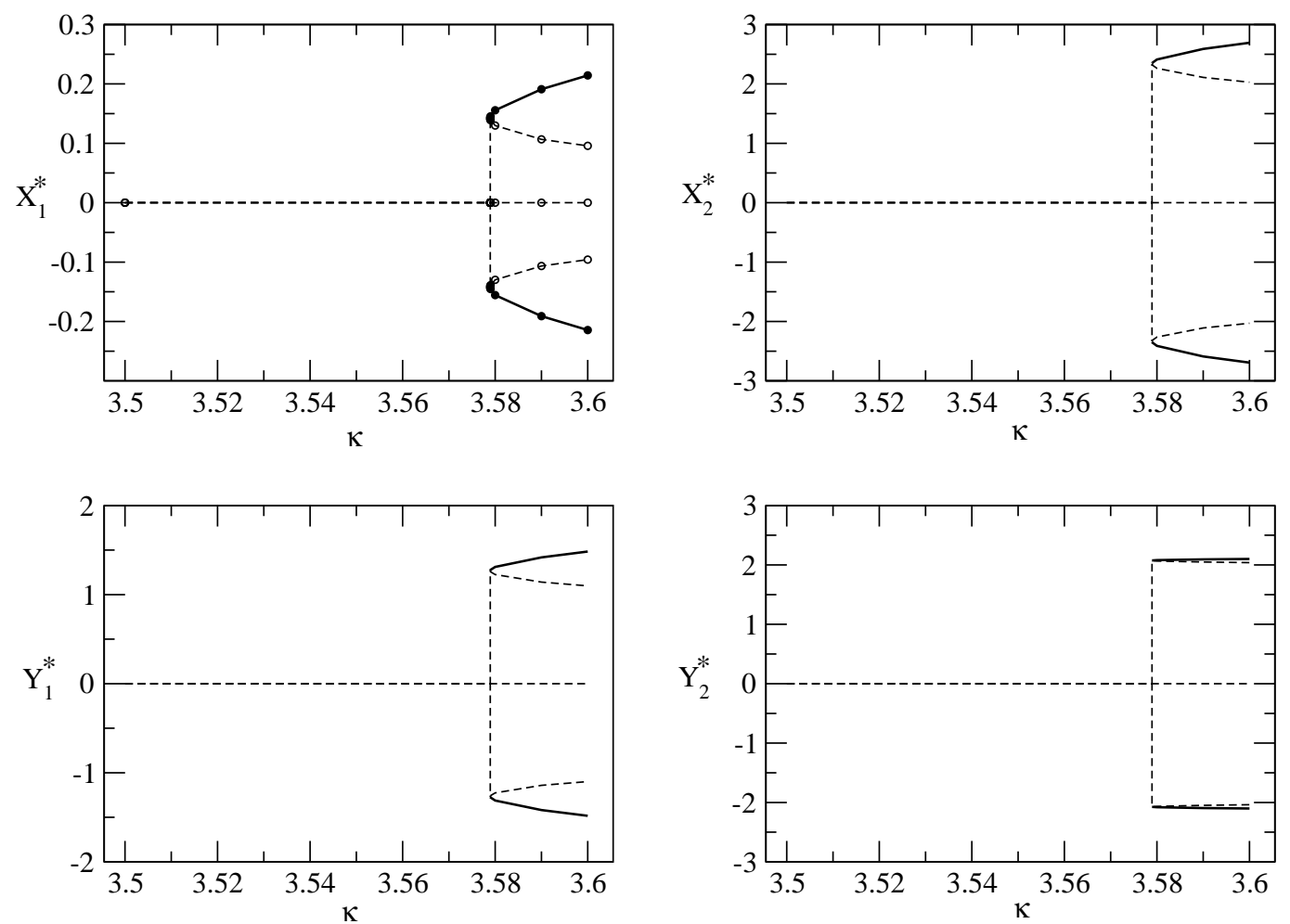

Figure 1. Bifurcation diagram that shows the appearance of new equilibria and their stability. The dashed lines correspond to unstable fixed points, while the continuous line depicts stable ones.

Analysis of Eq. (10) combined with Eq. (9) shows that, for $\epsilon \ll 2 \omega_{1}$ and $\epsilon \ll 2 \omega_{2}$, the only fixed point is $(0,0,0,0)$. For sufficiently large values of $\epsilon$, we obtain four new fixed points. The fixed points can appear only in pairs of stable-unstable points.

We now define $x_{1}=r_{1} \cos \left(\Phi_{1}\right), y_{1}=r_{1} \sin \left(\Phi_{1}\right), x_{2}=r_{2} \cos \left(\Phi_{2}\right), y_{2}=r_{2} \sin \left(\Phi_{2}\right)$, where $r_{1}^{2}=x_{1}^{2}+y_{1}^{2}$, $r_{2}^{2}=x_{2}^{2}+y_{2}^{2}$.

As in our model $\omega_{1} \gg \omega_{2}, a_{2}>a_{1}$, which implies $\left(x_{1}^{0}\right)^{2} \ll\left(x_{2}^{0}\right)^{2}$, from Eq (10) we obtain the equality:

$$
\omega_{2}=\epsilon \cos \left(\Phi_{2}\right) \sin (\Phi 2)
$$

This is equivalent to the equation:

$$
\frac{2 \omega_{2}}{\epsilon}=\sin \left(2 \Phi_{2}\right)
$$

As $\sin \left(2 \Phi_{2}\right) \leq 1$, this means that we have new solutions to the algebraic equations (9) only when

$$
\epsilon>2 \omega_{2} .
$$

Eq. (13) can give us a simple (and understandable) analytic estimate of the critical value for the bifurcation.

Fig. 2 shows a numerical simulation of the coupled oscillators (6). We can see how the oscillations evolve as $\epsilon$ is being increased by steps of 0.1 . In this way we have determined that, for the chosen values of the parameters, the oscillations die off for $\epsilon_{c} \sim 3.58$. We should compare this value with that obtained with the theoretical condition (13), from where we get $\epsilon_{c} \approx 4 \pi f_{2}=3.77$. Both critical values are remarkably close, differing by a 


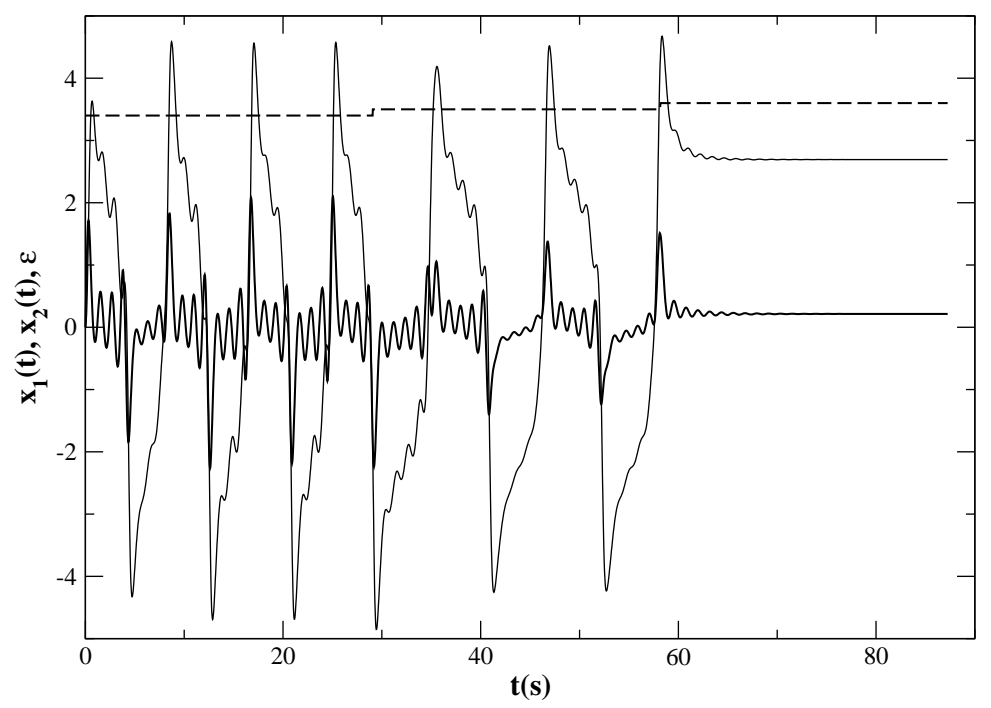

Figure 2. Time series of $x_{1}(t)$ (bold curve) and $x_{2}(t)$ from numerical simulations of the system (6). The parameters were: $f_{1}=1.1, f_{2}=0.3, a_{1}=0.5, a_{2}=1.0, \alpha_{1}=1$ and $\alpha_{2}=1$. The dashed line shows the step-wise variation of the coupling constant $\epsilon$.

small error accounted for by the assumptions of difference in order of magnitude of the natural frequencies in the system.

Using the same values of parameters as above, we also performed numerical simulations for negative $\epsilon$, and found that oscillation death occurs for $\epsilon \sim-2$, which agrees very well with the theoretical prediction (8).

\subsection{Five Oscillators}

\subsubsection{Numerical Simulations}

We have investigated numerically the algebraic equations

$$
\begin{array}{ll}
-x_{i} q_{i}-y_{i} \omega_{i}+\epsilon \sum x_{j} & =0 \\
-y_{i} q_{i}+x_{i} \omega_{i} & =0,
\end{array}
$$

where $q_{i}=\alpha_{i}\left(\sqrt{\left(x_{i}^{2}+y_{i}^{2}\right)}-a_{i}\right), i=1, \ldots, 5$. The solutions to these algebraic equations correspond to the fixed points of the dynamical system (1) when we have five coupled oscillators. We have found that, for sufficiently large values of $\epsilon$, the system possesses four additional fixed points (two stable and two unstable) as in the case of two coupled oscillators.

For instance, for $\epsilon=0.52$, the stable fixed points of the dynamical system are the following:

$$
\begin{aligned}
& x_{1}^{*}=0.022530 x_{2}^{*}=1.198176 x_{3}^{*}=2.550139 x_{4}^{*}=2.761457 x_{5}^{*}=2.489791 \\
& y_{1}^{*}=0.717314 y_{2}^{*}=1.861161 y_{3}^{*}=0.933874 y_{4}^{*}=0.292930 y_{5}^{*}=0.078571
\end{aligned}
$$

The points (15), and their negative symmetrical counterparts are asymptotically stable. These are the new attractors of the dynamical system, while the origin continues to be an unstable equilibrium. The calculation of the fixed point (15) is not a simulation. We have used the computer only as a means calculate of the values of the variables that are solutions to the algebraic equations (14). The concept of asymptotically stable fixed points is part of the theoretical understanding of the system.

We have also simulated the dynamics of the system for the given parameter values. The result of the simulation is that the phase trajectories are attracted to the asymptotically stable fixed points (see Fig. 3(A)). This is a manifestation of the phenomenon known as oscillation death. 

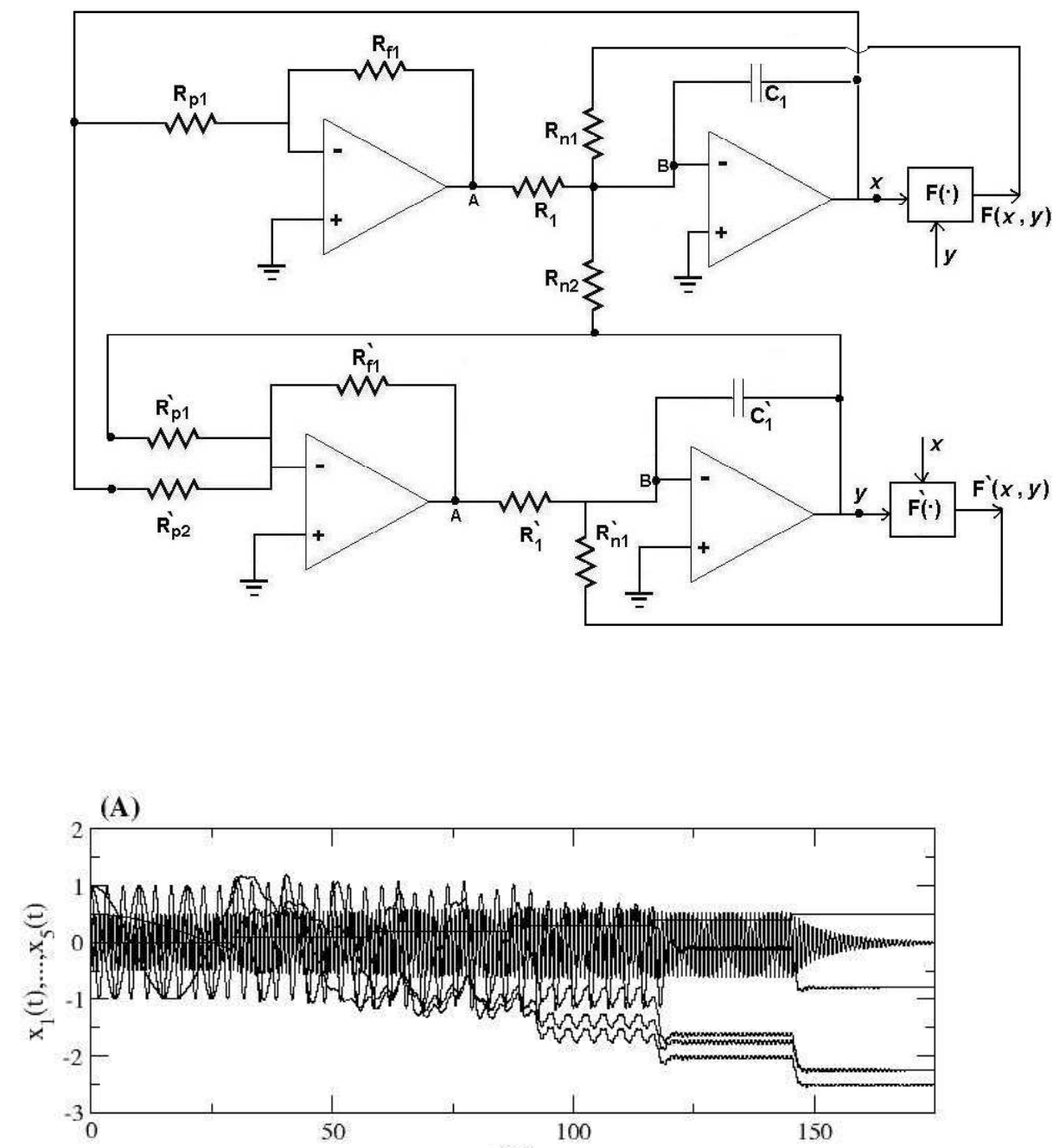

$\mathrm{t}(\mathrm{s})$

(B)

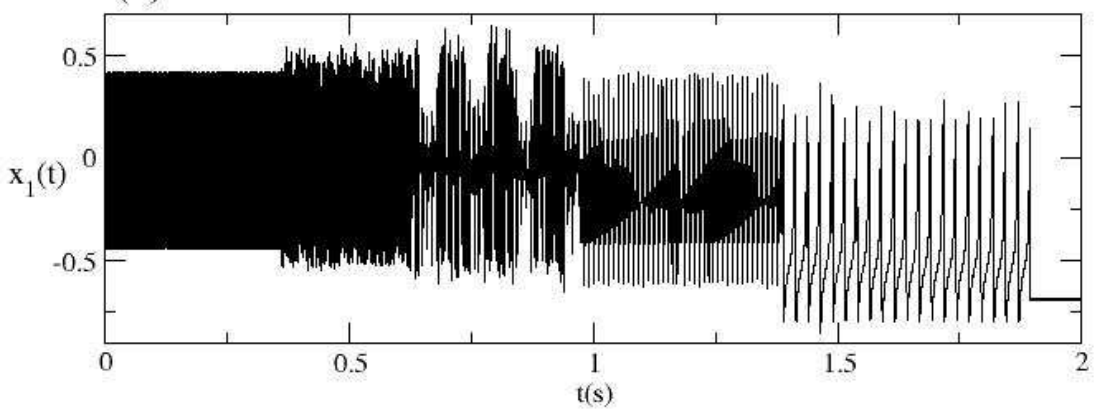

Figure 3. Top diagram: equivalent electronic circuit of a single Poincaré Oscillator (1). Time series (A), shows five functions $\left(x_{1}(t), \ldots, x_{5}(t)\right)$ as $\epsilon$ (bold steps) is increased, in the numerical simulation of system (14). Time series (B), shows the signal $x_{1}(t)$ in the experiments with five coupled oscillator circuits. 


\subsubsection{Real Experiments}

We performed experiments with analogue electronic circuits that show the phenomenon of oscillation death. The diagram of the circuit equivalent to the basic oscillator with equation (1), can be seen in the top picture of Fig. 3 . The blocks labeled $F(*)$ implement the nonlinear functions $F(x, y)=\frac{x}{10} \sqrt{x^{2}+y^{2}}$, and $F^{\prime}(x, y)=\frac{y}{10} \sqrt{x^{2}+y^{2}}$. In our experiments we coupled five of these Poincaré oscillator circuits using the mean field coupling approach.

The result of the experiments are shown for $x_{1}(t)$ in the time series (B) of Fig. 3. For comparison in the same figure, the time series (A), shows the numerical simulations of the same system of five coupled oscillators. In both figures we can clearly see the collapse of the oscillations to some non-zero constant values, when the coupling intensity $\epsilon$ reaches a critical value.

This demonstrates that oscillation death is a robust dynamical phenomenon that can be appreciated in real systems, when the conditions necessary for its manifestation are met.

\section{FREQUENCY VARIABILITY IN THE COUPLED OSCILLATORS}

In this section we will investigate several phase equations in order to get some insight on the frequency variability of nonlinear oscillators. We believe phase dynamics is the key approach to understand frequency variability.

First we will study some non-autonomous phase equations as the following:

$$
\dot{\Phi}=\omega_{0}+F(t) .
$$

These equations can be solved exactly:

$$
\Phi(t)=\Phi_{0}+\omega_{0} t+\int_{0}^{t} F(\tau) d \tau
$$

We will use different functions for the perturbation $F(t)$. The simplest case is that with no perturbation at all:

$$
F_{1}(t)=0
$$

Then we will analyze the case of periodic perturbation.

$$
F_{2}(t)=\epsilon \cos (\omega t)
$$

and quasiperiodic perturbation:

$$
F_{3}(t)=\epsilon\left(a_{1} \cos \left(\omega_{1} t\right)+a_{2} \cos \left(\omega_{2} t\right)+a_{3} \cos \left(\omega_{3} t\right)\right) .
$$

The following function

$$
F_{4}(t)=\epsilon \sin \left(B_{1} \sinh \left(a_{1} \cos \left(\omega_{1} t\right)+a_{2} \cos \left(\omega_{2} t\right)\right)+B_{2} \cosh \left(a_{3} \cos \left(\omega_{3} t\right)+a_{4} \cos \left(\omega_{4} t\right)\right)\right)
$$

has been shown recently to behave as a chaotic perturbation with some properties that resemble white noise. ${ }^{16}$

Finally, for $F(t)$ in Eq. (16) we will use the dynamics produced by a chaotic system:

$$
F_{5}(t)=\epsilon X_{1}(t)
$$

where $X_{1}$ is a component of the chaotic Lorenz system:

$$
\begin{array}{ccc}
\dot{X}_{1} & =10(X 2-X 1), \\
\dot{X}_{2} & = & 28 X_{1}-X_{2}-X_{1} X_{3}, \\
\dot{X}_{3} & =X_{1} X_{2}-(8 / 3) X_{3} .
\end{array}
$$



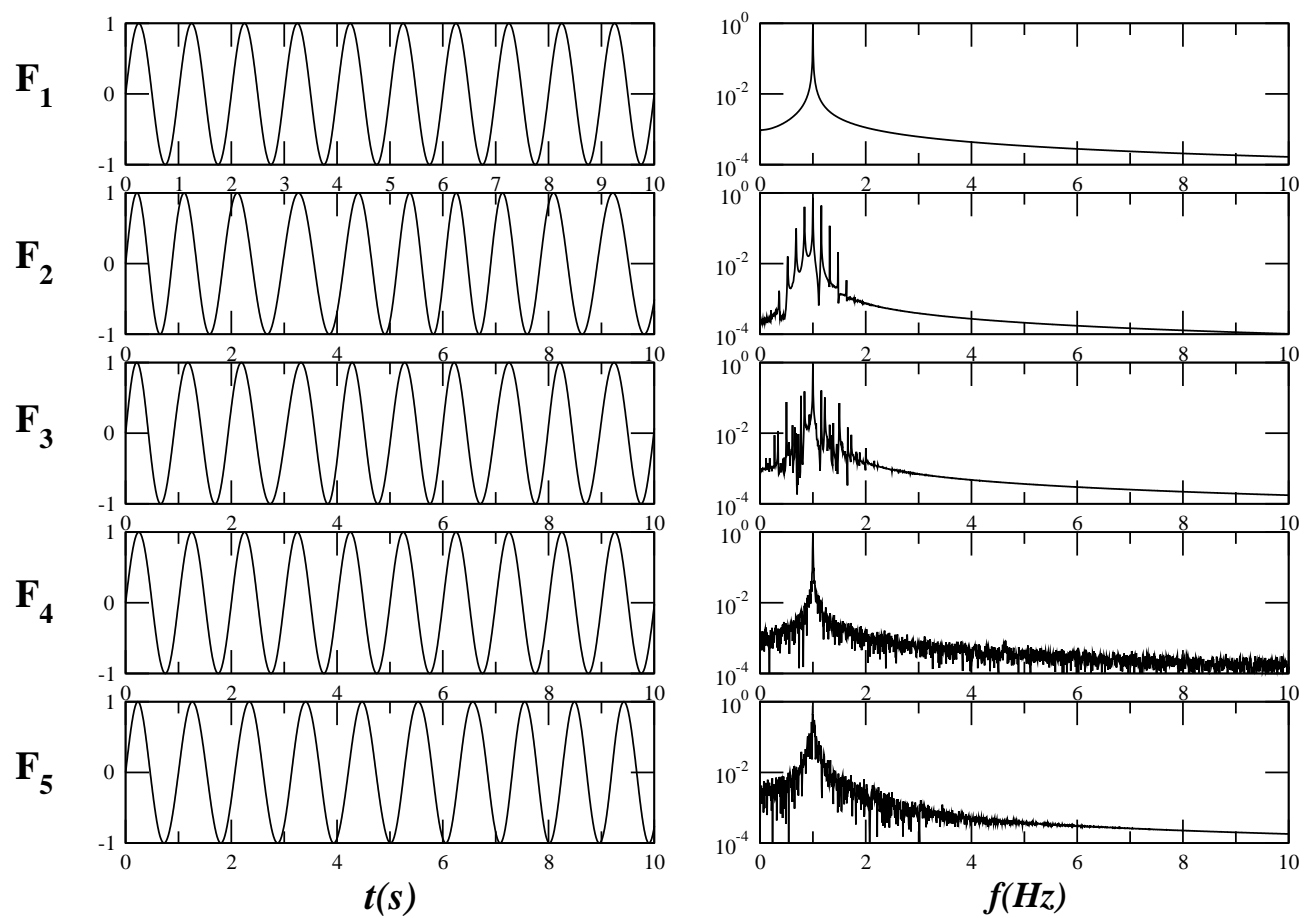

Figure 4. Left column: Time series of the solution, $X(t)=\sin (\Phi(t))$, of the phase equation (16) for different cases of the perturbation $F_{i}(t)$. Right column: The corresponding Fourier transforms of the functions $X(t)$.

The results of the Fourier transformation of the solutions to Eq. (16), and expressed as $X(t)=\sin (\Phi(t))$ are shown in the Fig. 4. As we can see, the best candidates for an explanation of the frequency variability are the cases given by Eq. (20) and Eq. (22). Eq. (20) corresponds to a quasiperiodic perturbation of the phase dynamics. On the other hand, Eq. (22) corresponds to a chaotic perturbation with a power-law spectrum.

Our cardiovascular model consists of a system of coupled autonomous oscillators. This model can be represented by the phase dynamics of some general autonomous dynamical systems, that describe their behavior. We now study some examples of these systems.

In general, the phase dynamics of an autonomous dynamical system can be described by a set of equations as the following:

$$
\begin{gathered}
\dot{\Phi}_{1}=\omega_{1}+\epsilon_{1} Q_{1}\left(\Phi_{1}, \ldots, \Phi_{n}\right), \\
\dot{\Phi}_{2}=\omega_{2}+\epsilon_{2} Q_{2}\left(\Phi_{1}, \ldots, \Phi_{n}\right), \\
\vdots \\
\dot{\Phi}_{n}=\omega_{n}+\epsilon_{n} Q_{n}\left(\Phi_{1}, \ldots, \Phi_{n}\right),
\end{gathered}
$$

However, sometimes a system like that given by Eq. (24) can be coupled to the amplitude dynamics of other dynamical systems.

Let us introduce the following set of equations that describe the phase dynamics of an autonomous dynamical 
Fourier Spectra

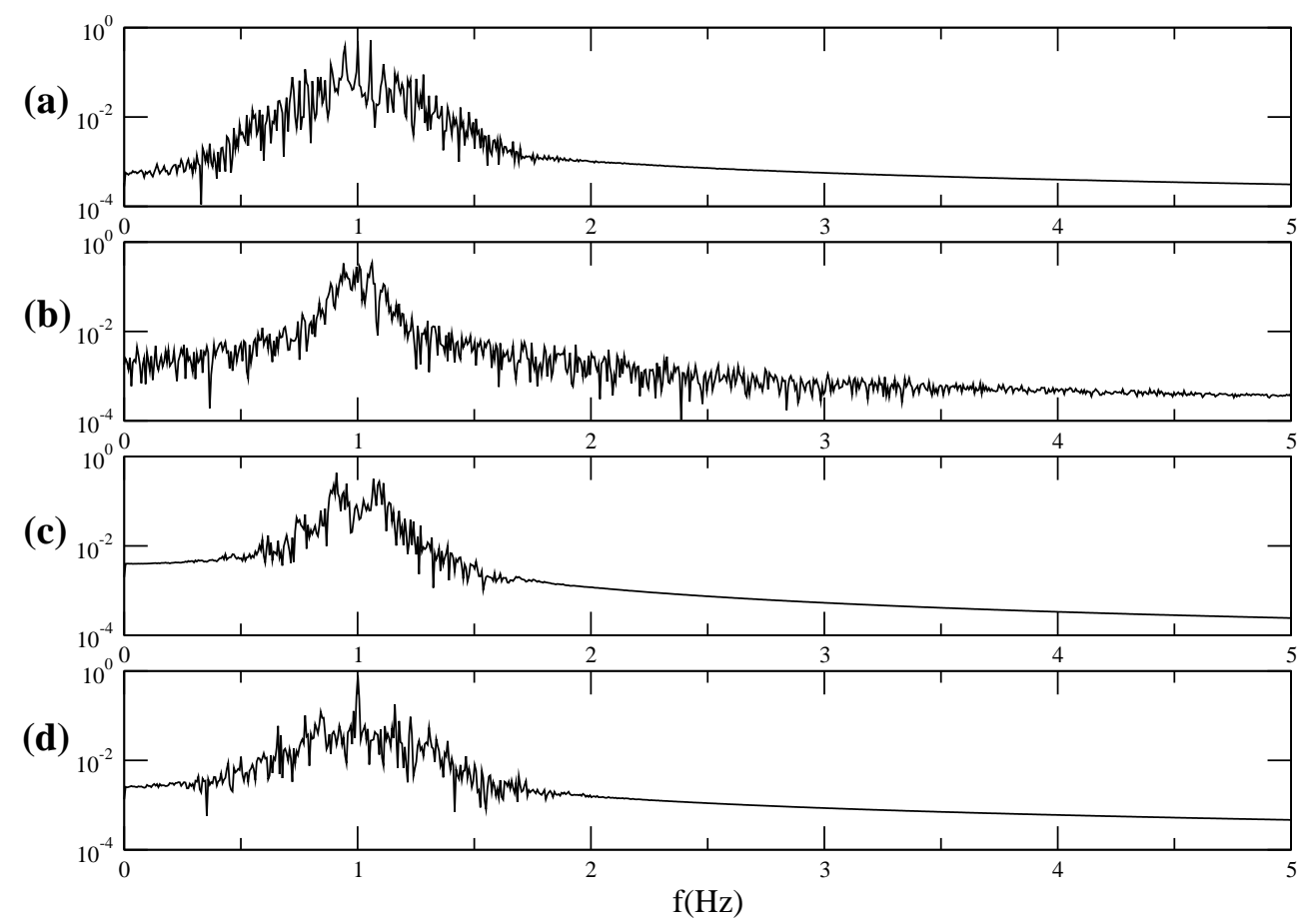

Figure 5. Fourier spectra of the dynamics generated by Eqs. (26)-(28). See the text for details.

system that represents a coupled oscillators model.

$$
\begin{aligned}
& \dot{\Phi}_{1}=\omega_{1}+\epsilon\left(a_{1} \cos \Phi_{1}+a_{2} \cos \Phi_{2}+a_{3} \cos \Phi_{3}\right), \\
& \dot{\Phi}_{2}=\omega_{2}, \\
& \dot{\Phi}_{3}=\omega_{3}, \\
& \dot{\Phi}_{4}=\omega_{4} .
\end{aligned}
$$

Analysis shows that, with the appropriate parameters, the dynamics of $\Phi_{1}(t)$, as seen through $X_{1}(t)=\sin \left(\Phi_{1}(t)\right)$, is equivalent to that produced by Eq. (16) with $F(t)$ as defined in Eq. (20). Thus, the frequency variability shown in Fig. 4 with perturbation $F_{3}(t)$ is the same as that obtained using the autonomous dynamical system (25).

We have also investigated other dynamical systems. The variability in the frequency of $X_{1}(t)=\sin \left(\Phi_{1}(t)\right)$ generated by the dynamical system:

$$
\begin{aligned}
& \dot{\Phi}_{1}=\omega_{1}+\epsilon\left(A_{2} \sin \Phi_{2}+A_{3} \sin \Phi_{3}+A_{4} \sin \Phi_{4}\right)+B_{2}\left(A_{5} \sin \Phi_{5}\right) \\
& \dot{\Phi}_{2}=\omega_{2} \\
& \dot{\Phi}_{3}=\omega_{3} \\
& \dot{\Phi}_{4}=\omega_{4} \\
& \dot{\Phi}_{5}=\omega_{5}
\end{aligned}
$$

is shown in Fig. 5(a). 
The next dynamical system possesses a chaotic (Lorenz) subsystem:

$$
\begin{aligned}
\dot{\Phi}_{1} & =\omega_{1}+\epsilon X_{2}+B A_{5} \sin \Phi_{5} \\
\dot{X}_{2} & =10(X 3-X 2), \\
\dot{X}_{3} & =28 X_{2}-X_{3}-X_{2} X_{4}, \\
\dot{X}_{4} & =X_{2} X_{3}-(8 / 3) X_{4}, \\
\dot{\Phi}_{5} & =\omega_{5}
\end{aligned}
$$

where $X_{1}(t)=\sin \left(\Phi_{1}(t)\right)$. The effect on its spectrum can be seen in Fig. $5(\mathbf{b})$.

Another example of an oscillator system coupled to a chaotic (Duffing) system is the following:

$$
\begin{aligned}
& \dot{X}_{1}=X_{2} \\
& \dot{X}_{2}=-\gamma X_{2}+X_{1}-X_{1}^{3}+f_{0} \sin \Phi_{3}, \\
& \dot{\Phi}_{3}=\omega_{3}, \\
& \dot{\Phi}_{4}=\omega_{4}+\epsilon_{1} X_{1}, \\
& \dot{\Phi}_{5}=\omega_{5} \\
& \dot{\Phi}_{6}=\omega_{6}+\epsilon_{2}\left(A_{3} \sin \Phi_{3}+A_{4} \sin \Phi_{4}+A_{5} \sin \Phi_{5}\right),
\end{aligned}
$$

where $\gamma=0.25, \omega_{3}=1$ and $f_{0}=0.3$. The spectrum of $X_{4}(t)=\sin \left(\Phi_{4}(t)\right)$ is depicted in Fig. $5(\mathbf{c})$, and that of $X_{6}(t)=\sin \left(\Phi_{6}(t)\right)$ in Fig. $5(\mathbf{d})$.

We have investigated several systems of type (24) that represent coupled oscillators models. The simple fact that the oscillators are coupled does not always lead to frequency variability like that shown in cardiovascular dynamics. Not even the fact that the coupling generates chaos is sufficient for explaining the frequency variability. Some examples of the investigated time series are shown in Fig. 5.

We believe the coupling of 4 or 5 oscillators is needed for observing the CVS frequency variability. The relationship between the frequencies is important. If the system is not chaotic, the influence of 3 or more oscillators on the cardiac oscillators is necessary. The coupling needs both linear and nonlinear terms. If a subsystem of the model is chaotic, the coupling of the cardiac oscillator with the chaotic system can produce the frequency variability, provided that the Fourier spectrum of the chaotic system possesses the right properties.

\section{CONCLUSIONS}

We have investigated a model of the cardiovascular system based on a system of coupled nonlinear oscillators.

We have demonstrated that different mechanisms are possible for the explanation of actual pathological or normal physiological behavior. For instance, sudden cardiac death or disease-induced changes in HRV can be explained in a deterministic way inside the framework of this model.

We have used theoretical and numerical techniques, and analogue electronic experiments as well. We have found that the phenomenon of oscillation death can occur in this model. There are two mechanisms for this effect. When the coupling strength $\epsilon$ is negative and is smaller than some critical value, amplitude death can occur. The zero-amplitude fixed point becomes asymptotically stable and the oscillations die to zero. This is a Hopf bifurcation scenario, in which the frequency of the oscillations remains almost constant while the amplitude slowly vanishes to zero.

In the second case, oscillation death happens when in the coupled system new fixed points appear in the place of the limit cycle, after $\epsilon$ has surpassed a positive threshold. In this case, the amplitude remains almost constant wile the frequency slowly vanishes to zero. This case corresponds to a saddle-node bifurcation, and is the scenario more likely to occur in actual coupled physiological oscillators.

The discussion of oscillation death is usually focused on the strength of the coupling coefficient. The common phrase is that "for large couplings, amplitude death will take place". However, once we have an analytical expression for the critical value (which will depend on other parameters), then we can predict the phenomenon when different parameter relations are changed. 
For instance, the analytical conditions given by Eq. (8) for oscillation death indicates that if the "amplitude" parameters $a_{1}$ and $a_{2}$ are decreased (in fact it is sufficient to decrease the largest of these parameters), the amplitude death can occur even if the coupling coefficient is fixed. The same can happen if the "excitability" parameter $\alpha$ is decreased beyond some critical value.

Another mechanism is the following. If $\epsilon>0$, and $\epsilon>\epsilon_{c}$, new asymptotically stable fixed points can appear. In this case, the oscillations die but the dynamical variables can take non-zero asymptotic values. We should remark that the critical value $\epsilon_{c}$ depends on the frequency of the second oscillator (in this case it is the lowest frequency). That is, even if we fix the coupling strength $\epsilon$, if the frequency of one of the oscillators is less than some critical value, then the oscillators can stop dead.

These results could be applied in areas from high technology electronics to research on heart attacks.

For instance, the phenomena related to sudden cardiac death or the pathologies produced by aging could be explained by effects like these. Furthermore, these findings could suggest that some medications or medical treatments that lead to changes in the parameters $\alpha, a_{i}$ or $\omega_{i}$, could reanimate a person in whom cardiac oscillation death has occurred.

Recently some authors ${ }^{8}$ have speculated that death by delay might be involved in arrhythmias of the cardiac pacemaker network. However, other authors ${ }^{17}$ suggested that this is impossible because the propagation delays are too short in these networks. However, the oscillation death produced by changes in parameters of the coupled oscillators of the whole cardiovascular system seem very probable. For instance, according to our results, changes in the respiratory frequency could affect the cardiac oscillator.

These results could be applicable also in coupled arrays of neurons in the brain.

In the second part of the paper, we have investigated frequency variability in coupled oscillators. First we have shown that if the phase dynamics is forced by quasiperiodic or chaotic perturbations, frequency variability very similar to that observed in real measurements can be obtained.

Furthermore, we have shown that this kind of dynamics can be generated in autonomous dynamical systems consisting of coupled oscillators.

Our investigation suggests that the coupling of 4 or 5 oscillators is needed for observing the measured CVS frequency variability.

If a subsystem of the model possesses some chaotic behavior, the coupling of the cardiac oscillator with some of the chaotic variable can produce the frequency variability provided the Fourier spectrum of the chaotic variable satisfy some conditions (e.g. $1 / f$ spectrum).

If the system is not chaotic, the influence of 3 or more oscillators on the cardiac oscillator is necessary.

\section{ACKNOWLEDGMENTS}

We gratefully acknowledge support by the Programme Alßan, EU, scholarship number E03D05224VE. And by the Engineering and Physical Sciences Research Council, The Wellcome Trust, the EC through FP6 and the BRACCIA programme.

\section{REFERENCES}

1. Y. Kuramoto, Chemical Oscillations, Waves, and Turbulence, Springer-Verlag, Berlin, 1984.

2. L. Glass and M. C. Mackey, From Clocks to Chaos: The Rhythms of Life, Princeton University Press, Princeton, 1988.

3. A. Pikovsky, M. Rosenblum, and J. Kurths, Synchronization - A Universal Concept in Nonlinear Sciences, Cambridge University Press, Cambridge, 2001.

4. D. G. Aronson, G. B. Ermentrout, and N. Kopell, "On the stability of coupled chemical oscillators," Physica D 41(3), p. 403, 1990.

5. G. B. Ermentrout, "Oscillator death in populations of "all to all" coupled nonlinear oscillators," Physica D 41(2), p. 219, 1990. 
6. G. B. Ermentrout and N. Kopell, "Oscillator death in systems of coupled neural oscillators," SIAM J. Appl. Math. 50(1), p. 125, 1990.

7. R. Mirollo and S. Strogatz, "Amplitude death in an array of limit-cycle oscillators," J. Stat. Phys. 60(1-2), pp. 245-262, 1990.

8. D. V. R. Reddy, A. Sen, and G. L. Johnston, "Time delay induced death in coupled limit cycle oscillators," Phys. Rev. Lett. 80(23), pp. 5109-5112, 1998.

9. D. V. R. Reddy, A. Sen, and G. L. Johnston, "Experimental evidence of time-delay-induced death in coupled limit-cycle oscillators," Phys. Rev. Lett. 85(16), pp. 3381-3384, 2000.

10. R. Herrero, M. Figueras, J. Rius, F. Pi, and G. Orriols, "Experimental observation of the amplitude death effect in two coupled nonlinear oscillators," Phys. Rev. Lett. 84(23), pp. 5312-5315, 2000.

11. A. N. Pisarchik, "Oscillation death in coupled non-autonomous systems with parametrical modulation," Phys. Lett. A 318(1-2), pp. 65-70, 2003.

12. Y. Zhai, I. Z. Kiss, and J. L. Hudson, "Amplitude death through a hopf bifurcation in coupled electrochemical oscillators: Experiments and simulations," Phys. Rev. E 69, p. 026208, 2004.

13. A. Stefanovska, Self-Organisation of Biological Systems Influenced by Electric Currents, PhD thesis, University of Ljubljana, Ljubljana, 1992.

14. A. Stefanovska and M. Bračič, "Physics of the human cardiovascular system," Contemporary Phys. 40(1), pp. 31-55, 1999.

15. A. Stefanovska, D. G. Luchinsky, and P. V. E. McClintock, "Modelling couplings among the oscillators of the cardiovascular system," Physiol. Meas. 22(3), pp. 551-564, 2001.

16. J. A. González, L. I. Reyes, J. J. Suárez, L. E. Guerrero, and G. Gutierrez, "Chaos-induced true randomness," Physica A 316, pp. 259-288, 2002.

17. S. H. Strogatz, "Nonlinear dynamics: Death by delay," Nature 394, pp. 316-317, 1998. 